\title{
Dal catalogo alla narrazione identitaria
}

\author{
Maria Serena Sapegno \\ Università di Roma «La Sapienza» \\ mariaserena.sapegno@uniroma1.it
}

\begin{abstract}
Il saggio delinea le caratteristiche principali dei diversi modelli di narrazione storico-letteraria (Crescimbeni, Tiraboschi, Foscolo) che si succedono in Italia nel secolo diciottesimo e costituiscono il retroterra politico-culturale delle scelte che presiederanno alla composizione della Storia desanctisiana.
\end{abstract}

Parole chiave: narrazione; storia; identità nazionale; primato.

\begin{abstract}
The essay outlines the main characteristics of the different models of literary-historical narration (Crescimbeni, Tiraboschi, Foscolo) which followed one another in Italy in the 18 th century and set up the political-cultural background that stands out in the composition of De Sanctis's Storia.
\end{abstract}

Keywords: narration; Storia; national identity; primacy.

È certamente nel Settecento, il secolo delle rivoluzioni, che vanno rintracciate le origini di molti tra i nuclei fondanti della modernità europea, e perfino della post-modernità, tra gli altri naturalmente anche l'inveterata abitudine al medesimo uso del secolo come categoria periodizzante.

In particolare mi interessa qui osservare come il Settecento sia il secolo nel quale lo strumento della narrazione va assumendo una importanza crescente e per certi versi nuova. Sia che si tratti di usarla nella costruzione di identità del nuovo individuo pubblico nelle numerose scritture autobiografiche di uomini illustri, sia che si voglia rappresentare attraverso invece la finzione, spesso pseudo-autobiografica, la vita e le avventure dei nuovi eroi, investiti del ruolo pionieristico di fondatori della società moderna, o ancora che si voglia affidare alla narrazione il compito di comunicare con particolare efficacia una nuova concezione filosofica. Si tratta in ogni caso di Istorie, una forma di racconto che si 
allontana sempre di più dal mito di tradizione antica e anche dalla narrazione fantastica legata al romance, per farsi invece chiave di lettura ed intervento sul presente, strumento di elaborazione e costruzione della memoria, individuale e collettiva. A guardar bene è un approccio non diverso da quello utilizzato nello stesso lasso di tempo allo scopo di costruire e rappresentare l'altra identità che va prendendo allora forma, quella delle nascenti 'nazioni'.

Infatti nei diversi paesi europei, accanto all'ingrossarsi del vivace moto riformatore che investe tanti aspetti della società, ferve anche durante il secolo un'attività di 'costruzione del passato'. Lo scopo di tale attività, nuova soprattutto per ampiezza di scala e ambizione di prospettive, è quello di contribuire al soddisfacimento di un bisogno crescente di identificazione che sembra essere una delle forme in cui si esprimono la strisciante crisi delle società di ancien régime e l'affermarsi di nuovi protagonisti, nonché l'affacciarsi di profondi conflitti che tendono a disgregare dall'interno la società. In questa prospettiva, l'attività erudita di accumulo di documenti di vario genere è vista da molti come base necessaria di qualsiasi esercizio culturale e di grande utilità per la definizione di un'identità nel presente, attraverso la proiezione delle sue radici in una memoria comune e condivisa.

Se tale mi sembra il quadro di riferimento generale in cui leggere anche le vicende italiane, è certo innegabile che il caso italiano presenti delle atipicità marcate, che la modernità giunge non a smussare ma semmai a sottolineare. Il secolo che nasce deve infatti subito registrare in Italia l'avvenuta pubblicazione (Roma 1698), seguita da diverse ristampe ed ampliamenti, di una Istoria della volgar poesia, ad opera dell'abate Giovan Maria Crescimbeni, primo Custode dell'Accademia dell'Arcadia. La stessa messa a fuoco dell'oggetto 'poesia volgare' non è, a quella altezza cronologica e in quell'ambiente culturale né scontata né banale. Va infatti osservato che tale focalizzazione corrisponde da un lato ad una certa idea di modernità e dall'altro ad un'interpretazione dell'Accademia in chiave 'nazionale' ed egemonica (interpretazione che avrà del resto un vasto successo). Nel termine 'Istoria' invece credo vada letta non tanto ancora un'ambizione propriamente narrativa, quanto almeno la premessa metodologica che ad una narrazione teoricamente presiede, quella cioè della necessità di una selezione dei materiali in base ad un giudizio di valore e a dei criteri, accompagnata da ipotesi di uno sviluppo e di una finalità.

In effetti, al di là della sua struttura macchinosa e ideologica, caratterizzata prevalentemente nel senso di un accumulo quantitativo, la Istoria del Crescimbeni esprime una convinzione. Il modo migliore per contribuire alla missione salvifica dell'Accademia, cioè sottrarre la cultura italiana al baratro nel quale è precipitata nel secolo appena concluso, sta precisamente nel restituire agli Arcadi, e attraverso di essi capillarmente a tutti i colti della penisola (sotto la guida degli Arcadi), il senso forte di una tradizione specifica: quella poesia in volgare viene pertanto ufficialmente consacrata da un lato come la base comune a tutti i letterati italiani e dall'altro come una inestimabile ricchezza di cui tornar fieri per riprendere un posto d'onore tra le nazioni civili. Un'identità poetica dunque, che fa perno sulla Roma papale e definisce la modernità 
in una continuità senza traumi, attraverso la ripresa di una poesia in volgare 'espurgata' dal cattivo gusto marinista. Si tratta in verità di un'operazione che avrebbe del paradossale, ma quel mettere insieme il diavolo (della poesia volgare) e l'acqua santa (della curia romana) è possibile ed auspicabile solo perché quella tradizione viene estenuata e privata di ogni carica conoscitiva e investita soltanto di un valore 'patrimoniale': è per questo che l'Arcadia riesce a farne la base del suo progetto egemonico che si estende di colonia in colonia attraverso i tanti confini dei diversi stati italiani, affratellando gli intellettuali in vesti poetiche e pastorali.

Un orizzonte piuttosto lontano certo da quel gruppo di intellettuali che negli stessi anni, tra Bologna e Milano per giungere a Modena, e ancora pochi anni dopo a Venezia, cominciando come arcadi, vestono anch'essi spesso abiti talari e sono animati da un analogo desiderio di voler chiamare a raccolta tutte le forze disponibili per "giovare alla buona letteratura d'Italia». ${ }^{1}$ Essi però si adoperano intorno a giornali eruditi, come fanno a Venezia i Zeno, Maffei e Vallisnieri, e inoltre coltivano rapporti con personaggi come Mabillon e Leibniz, o con la Royal Society di Londra, e rispondono ai nomi di Magliabechi, Bacchini, Muratori, Manfredi e Marsili. Si tratta in altre parole dell'incontro fecondo tra l'illustre tradizione dell'Istituto delle Scienze di Bologna e il metodo archivistico e filologico dei Benedettini francesi, e poi con la cultura veneziana, che apre le porte ad un rinnovamento metodologico e culturale nel quale cominciano a germogliare i grandi progetti muratoriani, portatori dei principi del razionalismo nelle scienze umane.

Non è già più il tempo dei grandi cataloghi alla Crescimbeni, utilizzati per dare il senso di una ricchezza patrimoniale con cui sentirsi forti e difendersi. Nella progettualità muratoriana si passa dal disegno interessante ma fantastico di una Repubblica Letteraria d'Italia, ancora memore dell'antico predominio romano e volta «a servir meglio la gloria dell'Italia», ${ }^{2}$ ad un piano di lavoro molto concreto, quello che nei Rerum Italicarum Scriptores intende mettere insieme tutte le sparse fonti e i documenti, necessari ad una storia unitaria d'Italia, attraverso una mobilitazione straordinaria e inedita delle migliori energie intellettuali del paese, ben al di là delle schiere degli Arcadi «per gloria delle loro patrie e per vantaggio della repubblica letteraria d'Italia». ${ }^{3}$ Analoghe raccolte di fonti esistevano già per gli altri paesi europei, messe insieme spesso tra fine Cinquecento e Seicento, nel clima culturale legato alla Riforma.

Il motto virgiliano dell'impresa chiarisce bene che siamo ora davanti ad una nuova 'narrativa' che si pone simbolicamente come erede di una lunga e dolorosa vicenda ma allo stesso tempo rilancia sulla modernità, con una comples-

1. Così recita la prefazione al primo numero dell'edizione stampata a Parma nel 1686 dell' importante giornale erudito italiano, il Giornale de'Letterati d'Italia, uscito inizialmente a Roma e, dopo Parma, trasferito a Modena dal suo editore Benedetto Bacchini.

2. Lamindo Pritanio, Primi disegni della Repubblica Letteraria d'Italia, in Ludovico Antonio Muratori, Opere, a cura di Giorgio FalCo e Fiorenzo Forti, Milano Napoli: Ricciardi, 1964, vol. 44, t. I, p. 185.

3. ID., Epistolario, in Opere, cit., t. II, p. 1879. 
sità e una profondità nuove, un'immagine forte e densa di significati diversi: ANTIQUAM EXQUIRITE MATREM.

Esiste inoltre la famosa lettera nella quale Muratori spiega il suo progetto, ed è rilevante notare come sia indirizzata a quel medesimo conte di Porcìa che di lì a qualche anno si farà personalmente promotore di un'iniziativa assai significativa presso tutti i grandi personaggi del tempo, primo fra tutti Vico, perché scrivano le loro autobiografie a scopo esemplare interno ma anche come immagine all'estero dell'Italia. Scrive nella lettera Muratori: «In mia gioventù altro non aveva io in testa che antichità greche e romane. Per lo contrario mi facevano male agli occhi le fortune dei secoli susseguenti, la loro storia, i loro scrittori, riti, costumi e imbrogli, trovando io dappertutto del meschino, del barbaro (e infatti non ne manca), e parendo a me di camminare solamente per orride montagne, per miserabili tuguri e in mezzo ad un popolo di fiere. Laonde se mi capitava tra le mani qualche storia od operetta di quei rozzi secoli io neppur la degnava di uno sguardo. Mi rido ora di me stesso». ${ }^{4} \mathrm{Ed}$ in effetti può ridere perché ha scoperto una vera miniera di documenti e di informazioni inedite che, messe insieme, gettano una luce interamente nuova sulla storia d'Italia, ma anche perché è riuscito a coinvolgere un numero inimmaginabile di collaboratori, inverando così il suo progetto di unificazione intellettuale.

Ma qui interessa soprattutto il pieno riconoscimento di un profondo mutamento di prospettiva con la caduta del paradigma classicista e la conseguente entusiastica scoperta di un'altra storia, quella dell'Italia dei tempi di mezzo. Si tratta di un'altra e nuovissima narrativa identitaria, sottolineata dalla ripresa della figurazione materna, di cui non sfuggiranno le implicazioni emotive e morali: "quasi Italia, parens nostra, eadem sempre non fuerit ac sit tam sub Romanis rerum dominis, quam sub Langobardis, Francis atque Germanis». 5 La condanna intellettuale, ma anche morale, nei confronti degli umanisti per l'idolatria classicista e per il disprezzo delle culture di mezzo è molto netta e rappresenta una vera e propria cesura, una svolta.

Naturalmente, dal momento in cui viene abbandonata l'opposizione civiltà/barbarie, dalla storia delle diverse "patrie», nel loro differenziarsi progressivamente nell'incontro con le altre culture dei popoli sopraggiunti e nel loro lasciare testimonianze tanto diverse anche nella lingua scritta dei documenti, l'immagine della "Italia, parens nostra" che si va presentando agli occhi di Muratori e dei suoi tanti collaboratori è sempre più ricca e variegata e richiede l'acquisizione e l'uso di nuovi e più elastici strumenti di analisi.

Bisogna partire da questo retroterra per iniziare a valutare appieno le caratteristiche dell'iniziativa di Girolamo Tiraboschi e comprenderne le scelte.

Se per un verso infatti il dotto gesuita è grande ammiratore del Muratori, gli succede come bibliotecario lavorando nella medesima Biblioteca Estense, ne coltiva la memoria e si pone esplicitamente in continuità con lui, per altri non

4. Ludovico Antonio Muratori, Lettera al Porcìa, in Opere, cit., t. I, p. 26-27.

5. ID., Antiquitates Italicae Maedii Aevi in Opere, cit., t. I, p. 588. 
vuole o non può comprendere appieno la portata delle sue scelte e soprattutto dare seguito e sviluppo alle potenzialità in esse implicite. Infatti la passione erudita e storiografica lo conduce a raccoglierne la sfida e, attraverso la sua monumentale e ricchissima Storia della letteratura italiana (1772-1782), ad estenderla alla costruzione della prima grande storia della cultura italiana, condotta con spirito filologico dagli albori della civiltà fino quasi ai suoi giorni. Inoltre la scelta, in sé originalissima, di documentare con la maggior completezza possibile le tracce delle diverse culture che si sono succedute sul suolo italiano, si può leggere come una tacita accettazione della correzione muratoriana: nel corso di un tempo lunghissimo, l'antica terra madre ha generato dal suo seno popoli e culture diverse, delle quali tutte spetta allo storico dar conto. E il nostro storico lo fa, dagli Etruschi al Seicento, con una accuratezza ed una completezza che rendono la sua opera un repertorio, ancora proficuamente consultabile, di notizie ed informazioni preziose su una grande varietà di aspetti dello sviluppo della cultura umana in territorio italiano. Certo paradossalmente è proprio quella dimensione storica della complessità culturale così genialmente aperta da Muratori, e che offre al lettore moderno spunti di sorprendente interesse, a restare invece del tutto muta per il dotto gesuita: il rincorrersi di tanti popoli diversi sullo stesso territorio appare infatti piuttosto fine a se stesso e in definitiva vano.

Eppure la stessa narrazione erudita non può bastare alle esigenze dei tempi che, nonostante tutto, il gesuita ha ereditato e fatto sue: pertanto alla struttura di impianto enciclopedico Tiraboschi intreccia una griglia di valori, derivati dalle correnti dell'Illuminismo italiano e condivisi anche da Muratori, identificati nella coppia oppositiva progresso/declino, (articolata poi in molte altre opposizioni come luce/tenebre, ragione/superstizione, buon gusto/cattivo gusto ecc.) sulla base dei quali cerca risposta alla fondamentale domanda sulle cause del declino delle scienze in Italia.

Proprio nello sforzo di rispondere a tale domanda egli ricorre preliminarmente, già nel primo tomo dell'opera, ad una divisione concettuale dalle conseguenze rilevanti, quella tra scienze ed arti liberali e, ancor più profondamente tra 'vero' e 'bello': «Nelle scienze v'ha luogo all'errore, finché esse non son giunte alla lor perfezione, cioè finché non è scoperta ed accertata la verità. Ma quando ciò accada, parmi che non vi sia luogo a decadimento. Ma non così vuol ragionarsi delle arti liberali che hanno il bello per il loro primario oggetto. Questo consiste nella unione, nella distribuzione, nell'ordine, nell'espression delle parti; e quando in ciò arrivi a quella perfezion che costituisce il bello, il volere avanzarsi oltre è il medesimo che dare addietro. Nelle arti liberali solo adunque e nel loro progresso ha luogo la linea curva, nella quale, ove uno sia giunto alla più alta cima, non può andare oltre senza ricadere al basso». ${ }^{6}$

La distinzione e la netta e irreparabile separazione sono interessanti da diversi punti di vista. Intanto non è certo banale l'insistenza sul progresso e l'apertura alle Scienze naturali che consente al gesuita di includere tra i suoi

6. Girolamo Tiraboschi, Storia della letteratura italiana, Milano: [Classici Italiani] 1822-26, [Modena 1772-1782] t. I, libro III, capo II. 
eroi Galileo Galilei. Infatti la sua originale definizione del Seicento come secolo ancipite, da un lato del Marino e dall'altra di Galileo e della nuova scienza, sarà ripresa da molti, e in particolare da De Sanctis.

Risulta molto chiara anche l'adesione al paradigma classicista, pur nel delinearsi di una nuova gerarchia che subordina il Bello al Vero scientifico oltre che metafisico. Eppure tale rinnovata fede nel modello classicista del Bello non deriva all'autore da una passiva accettazione della tradizione classicista italiana, ma ne costituisce piuttosto una nuova interpretazione. Mentre infatti, dal Duecento in avanti, lo storico ricostruisce la storia della cultura italiana per scansioni secolari, dando uno spazio rilevante ma non dominante allo specifico letterario, si va facendo via via più chiara quale interpretazione egli consideri necessaria alla sua impresa e quale ne sia lo scopo prioritario. Innanzitutto la venerazione verso Muratori non giunge a fargli accettare davvero la rivoluzionaria attenzione da questi dedicata alla cultura dei tempi di mezzo: se Muratori guardava con una risata di indulgente riprovazione alla propria giovinezza infatuata del modello classicista, che condannava all'oscurità otto secoli di cultura italiana, Tiraboschi non ci trova nulla da ridere, anzi. La sua narrazione infatti riprende l'interpretazione umanistica per rilanciarla su basi radicali: gli umanisti, sulla scorta del "gran Petrarca» latino, («il primo che gittandosi tra le tenebre, onde ogni cosa era miseramente ingombrata, cercasse di richiamare a nuova vita quegli uomini illustri dell'antiquità, senza la scorta de'quali appena poteasi sperare di dissiparle») ${ }^{7}$ hanno recuperato la civiltà (messa in salvo dall'operosità dei monaci) e costituiscono il cuore forte della cultura italiana, il contributo unico e incalcolabile che essa ha dato al resto d'Europa.

Si va così delineando una precisa idea di 'primato', quella che oppone la cultura italiana, in quanto collettivo operoso di studiosi, eruditi e bibliotecari (che si reincarna poi nella stessa Compagnia di Gesù, 'ingiustamente' oggetto di critiche contemporanee tanto severe da decretarne la chiusura) agli altri, che spesso si presentano nelle vesti minacciose dei 'barbari'. Nel dare risposta alla domanda sulle cause della decadenza delle scienze infatti, Tiraboschi identifica senza problemi nella presenza degli stranieri e nella loro influenza corruttrice, sia la causa di decadenza della letteratura latina («dopo la morte di Augusto cominciò Roma ad essere più assai che prima inondata da popoli stranieri (...) Con ciò a me pare che probabilmente si spieghi non solo la rozzezza dello stile di quegli che erano stranieri, ma di quegli ancora, a'quali il parlare latino era natio. Questi frammischiati co'barbari (...) ne apprendevano la maniera di favellare, ne adottavano le parole, vestivano i difetti del loro stile») ${ }^{8}$ che naturalmente quella della vittoria del "cattivo gusto" in tempi più recenti. Tale è la passione ideologica anti-straniero per la difesa del primato italiano da spingerlo a prendere esplicitamente le distanze dalla 'svolta' muratoriana ( Sembra che il dottissimo Muratori avesse una singolare predilezione per questi Barbari. Egli abbraccia ne'suoi Annali ogni occasione che gli si offra a mostrare che

7. ID., Storia della Letteratura Italiana, cit., t.V, libro I, capo IV, par. v, p. 154.

8. ID., Dissertazione preliminare, in Storia della letteratura Italiana, cit., t. II, par. xxxi, p. 46-48. 
essi non eran poi né così barbari né così crudeli, come comunemente si crede»). ${ }^{\text {? }}$

Coerentemente a tale ricostruzione, del resto, se da un lato Tiraboschi non è in grado di percorrere davvero la nuova via aperta da Muratori, via che uscirà largamente sconfitta nella sua audacia rivoluzionaria, dall'altro anche nelle scelte del gesuita è l'intera narrazione tradizionale ad uscirne profondamente modificata, attraverso un cambiamento della sua base identitaria: il canone poetico italiano che aveva costituito l'asse portante della tradizione rinascimentale e sul quale si era voluto costruire il principato letterario italiano, recede ora in posizione esornativa per motivi sostanziali di valutazione ideologica e culturale.

Se infatti Petrarca resta modello di perfetta poesia e va accettato (e perdonato) quando «sfoga pietosamente la sua amorosa passione» ${ }^{10}$ certo altra cosa è «quando levasi più sublime e prende più nobili oggetti a scopo delle sue rime». ${ }^{11}$ Ancora più esplicitamente, dopo la rivoluzione degli umanisti che praticavano «occupazioni più degne d'uom dotto, e la Poesia Italiana parea in confronto ad esse un fanciullesco intrattenimento ${ }^{12}$ il Cinquecento viene presentato per contrasto come letterariamente sbiadito ma illuminato dal Concilio di Trento, dalla Compagnia di Gesù e dalla filosofia.

Tiraboschi consegna quindi ai suoi contemporanei, e ai posteri, un modello forte e autorevole, destinato a lunga fortuna: una narrazione storica accurata e fortemente marcata dalle domande e dalle urgenze del presente, inserita nel dibattito contemporaneo e volta a fornire le basi culturali di un'identità nazionale unitaria. Una storia erudita ma militante che, pur cercando di fornire giudizi equanimi tenendo conto dei diversi aspetti della cultura che va ricostruendo, e tentando di storicizzare i giudizi di valore, compie tuttavia le sue scelte ed è orientata da valutazioni di ordine ideologico e morale, proprio come rivendicato ripetutamente dal suo estensore. La spinta a restaurare l'orgoglio nazionale del primato, innanzitutto, ma anche un'idea di cultura come impegno per il progresso delle scienze e il senso etico di tale scavo storico.

Sono questi tutti elementi che verranno ripresi nella sua Storia da De Sanctis che lo definirà «il Muratori della letteratura», apprezzandolo e allo stesso tempo sottovalutando le profonde differenze tra i due impianti, mosso da una fortissima urgenza che però non gli impedirà di concludere la sua Storia con una valutazione dei suoi tempi che suona omaggio implicito al metodo del gesuita: «I sistemi sono sospetti, le leggi sono accolte con diffidenza, i principi più inconcussi sono messi nel crogiuolo, niente si ammette più che non esca da una serie di fatti accertati. Accertare un fatto desta più interesse che stabilire una legge». ${ }^{13}$

Giudizio in verità assai vicino a quello che apparirà di lì a poco sulle pagine programmatiche del primo numero del Giornale storico della letteratura

9. ID., Storia, cit., t. III, libro II, capo I, par. iv, p. 129.

10. Ibid., t. VI, libro III, capo II, par. xxxv, p. 815 .

11. Ibid.

12. Ibid., capo III, p. 1226.

13. Francesco De SANCTis, Storia della letteratura italiana, Bari: Laterza, 1925, vol. II, p. 417. 
italiana: «che cosa sono, (...) dopo quella del Tiraboschi, (...) le storie della nostra letteratura? $\mathrm{O}$ esposizioni superficiali e manchevoli, o sintesi più o meno geniali in cui, più assai che allo studio diretto dei fatti, si badò ad alcuni preconcetti estetici, politici, filosofici, con l'aiuto de' quali si pretese di interpretare e ordinare fatti male sceverati e mal noti, ossia di ricostruire sistematicamente la storia». ${ }^{14}$

La Storia desanctisiana e quella tiraboschiana sembrano in queste parole percepite ormai come modelli opposti e inconciliabili, tra i quali preferire senza dubbio quello del gesuita in quanto farebbe a meno di una struttura sistematica, di un ordinamento narrativo.

Era stata questa, dell'opposizione inconciliabile tra i due modelli, anche l'opinione del Foscolo, che era però severissima, considerando all'opposto la mancanza di una narrazione un difetto imperdonabile, pur se non scevra di rispetto e ammirazione per il formidabile lavoro di scavo, accertamento ed ordinamento di materiali che risultava evidente ad apertura e consultazione dei monumentali volumi tiraboschiani.

In particolare Foscolo osserva acutamente che «de' letterati italiani raramente il Tiraboschi mi mostra quanto giovarono o quanto nocquero alla loro arte ed al mondo. (...) Vero è che nelle Dissertazioni preliminari il nostro storico intende di provvedervi, ma (...) se la storia senza filosofia non è che serie cronologica d'avvenimenti, le disquisizioni critiche senza avvenimenti non sono mai storia». ${ }^{15} \mathrm{Da}$ storico della letteratura, ma soprattutto da scrittore tutto dentro la contemporaneità dei fatti letterari, e da poeta, Foscolo coglie la profonda debolezza della ricerca del gesuita da un lato nell'incapacità di costruire una narrazione che renda conto dello sviluppo dell'arte poetica, della letteratura, nel tempo e attraverso i contributi dei singoli, dall'altro nello scollamento che perciò rimane tra i medesimi 'fatti letterari' e la trattazione storica.

Sappiamo che, pur senza giungere mai a completare il suo disegno di storia letteraria, Foscolo si dedica a lungo e con grande assiduità allo studio della letteratura italiana dal punto di vista storico-critico e lascia un materiale ricchissimo ed affascinante pur se in parte frammentario. Per lui è proprio la narrazione che deve costruire il senso del percorso e l'identità culturale italiana ha un filo rosso semplice e fortissimo: la lingua. La lingua letteraria gli appare la chiave per ricostruire le epoche della letteratura italiana, presa in esame perché "per essere la più antica e copiosa tra tutte le moderne, può contribuire più efficacemente alla storia della civiltà e della critica letteraria». ${ }^{16}$ Da questa angolazione, fondata nel presente di chi si è trovato a fare i conti con la mancanza di una lingua moderna nella quale poter scrivere, molti snodi appaiono cruciali, le domande sui fatti letterari, sui testi, si radicano nella storia civile e la dannosa separazione imputata a Tiraboschi viene meno.

14. Giornale storico della letteratura italiana, «Programma”, vol. I (1883), 1, p. 2.

15. Ugo Foscolo, Edizione Nazionale delle Opere, Firenze: Le Monnier, 1933, vol. VII, p. 49.

16. Ibidem, vol. XI, p. 99. 
La chiave della lingua e l'intelligenza della poesia aprono le porte di una storia letteraria integralmente nuova, fatta di testi e di uomini, fatta di problemi ad un tempo stilistici e storici, di opere letterarie scritte per rispondere a questioni che si ponevano ad uomini inseriti in un contesto di passioni personali e collettive, potremmo dire politiche in senso generale. Si tratta quindi di una storia militante su piani molteplici poiché la passione politica e quella poetica, insieme ai tempi nuovi nei quali si trova a vivere e a pensare, consentono a Foscolo di cogliere con profondità inedita intrecci mai davvero compresi prima.

Per cominciare con Dante, egli è il primo a mettere in relazione feconda il teorico della lingua con il poeta, a riconoscere la visione profetica non solo del poeta-teologo, ma anche dell'intellettuale che voleva «avvezzare tutti gli Italiani a comunicarsi a vicenda le leggi, la storia patria, i pensieri e gli affetti con una lingua scritta (...) confondendo e fondendo (...) tutte le parole e le locuzioni (...) più atte a comporre la lingua letteraria e generale della nazione». ${ }^{17}$

Proprio la stessa raffinata percezione che lo conduce a esaltare Petrarca come colui che porta a perfezione la lingua poetica in quanto codice precipuo delle passioni del soggetto.

Ma è invece la passione politica che gli fa cogliere le conseguenze devastanti del fatto che se "la lingua italiana nacque e crebbe dalla libertà popolare» eppure la lingua letteraria comune fu lingua sempre scritta e mai parlata e pertanto «la nazione in generale non ne ricavò mai profitto». In questa narrazione unitaria tutta incentrata sulla lingua volgare non può naturalmente trovare posto alcuna celebrazione dell'Umanesimo, fenomeno nella valutazione del quale anzi si palesa una forte novità nel punto di vista, quella che considera precisamente l'adorazione dell'antico come parte del più generale stato di servitù del paese e come una delle concause della catastrofe italiana: «gli scrittori contemplavano oziosamente l'antica Roma ed Atene più che l'Italia; e scrivendo in latino, andavano riducendosi più sempre a comunità diversa al tutto dalla nazione». ${ }^{18}$

$\mathrm{Si}$ avverte in questo sguardo dello storico una lucidità del tutto nuova e fuori dall'ordinario, data probabilmente oltre che dalla particolare esperienza politica e culturale, anche dalla sua peculiare posizione di interno/esterno alla situazione italiana e certo dal fatto che proprio in quegli anni, (per dirla con Bollati) «un processo irreversibile era iniziato» e ne erano «stati designati i principali interessati». ${ }^{19}$

Sono gli stessi anni in cui circola con clamore e successo la Littérature del Sismondi, che batte su molti dei tasti già toccati da Foscolo e invita gli italiani ad assumere una narrazione identitaria decisamente moderna. Ma sono anche gli anni in cui gli intellettuali italiani, romantici o classicisti che fossero, dovettero al contrario via via attenuare le proprie differenze politico-culturali per

17. Ibid., vol. X, p. 313.

18. Ugo FosColo, Edizione Nazionale delle opere, cit., vol. XI, I, p. 227.

19. Giulio Bollati, L'Italiano. Il carattere nazionale come storia e come invenzione, Torino: Einaudi, 1983. 
partecipare alla stessa impresa, un'occasione storica che probabilmente costò il prezzo di una mancata chiarezza, che in verità nessuno desiderava davvero, e non solo perché avrebbe significato scelte che apparivano intempestive e fonte di divisione. Sono gli anni in cui sarà l'editoria a Milano (Classici italiani) e poi a Firenze (la Biblioteca nazionale di Le Monnier) a mettere insieme, sotto l'egida della bella letteratura e della lingua, testi di autori che non erano mai stati sugli stessi scaffali, secondo una logica ancora una volta 'patrimoniale'.

Alla fine di questo periodo, per quanto caotico, pur sempre operoso e stimolante, sarà De Sanctis a scegliere invece in tale serbatoio confuso una linea narrativa e interpretativa che poi prevarrà nella nuova Italia. Forse perché anche lui come Foscolo aveva fatto l'esperienza dell'esilio, di quella acquisizione dall'esterno di uno sguardo d'insieme da cui raccontare agli stranieri la cultura italiana, de Sanctis dimostrerà una straordinaria sintonia con la sensibilità e l'approccio foscoliani. Rispetto alla militanza erudita e 'umanista' di Tiraboschi, infatti, Foscolo aveva saputo cogliere la necessità tutta nuova di forgiare un'identità nazionale che accentuasse la modernità della tradizione letteraria italiana, quella sua nascita nell'età della libertà popolare già ai livelli altissimi delle 'tre corone'. Ma allo stesso tempo aveva saputo trasmettere per primo l'urgenza e l'inevitabilità di un riscatto politico e morale cui la letteratura stessa poteva contribuire costruendo, attraverso l'edificazione di un nobile passato comune, il senso di una identità forte che aiutasse a superare le miserie del presente e soprattutto le profonde differenze tra le diverse culture della penisola.

La storia si incaricherà poi di dimostrare come si trattasse ancora una volta di una generosa illusione, o meglio della ripresa di una scena già vista e destinata a ripetersi innumerevoli volte: invece che della ricostruzione di una storia complessa e articolata di esperienze differenziate, si tratterà in buona sostanza di quella funzione di 'supplenza' esercitata da parte degli intellettuali che è sempre stata allo stesso tempo sintomo e concausa della debolezza italiana. E resta significativo il fatto che in definitiva a Tiraboschi, e al suo immenso lavoro di archivio della variegata produzione culturale della penisola, si sia poi per tanti versi tornati di recente, anche grazie alla consapevolezza crescente che la narrativa ideologicamente forte, che si era ritenuta necessaria al riscatto e alla costruzione di una identità comune, aveva però appunto trascurato molto di ciò che caratterizza la specificità e l'unicità della tradizione culturale del sistema Italia.

Nel raccogliere dunque l'eredità non di quella prima storia nazionale della letteratura, quanto della narrativa foscoliana, incompiuta ma brillante e appassionata, de Sanctis intende la propria impresa come misura temporanea da destinarsi alla scuola, ma la sua Storia costituirà invece la risposta ideale ad un'esigenza identitaria che finirà per prevalere su ogni altra considerazione, restando il capolavoro del suo genere e il modello implicito della maggioranza dei testi scolastici, ma sacrificando all'urgenza del fine la complessità contraddittoria delle diverse tradizioni italiane. 